\title{
Direct Observation of Magnetic Metastability in Individual Iron Nanoparticles
}

\author{
Ana Balan, ${ }^{1}$ Peter M. Derlet, ${ }^{2}$ Arantxa Fraile Rodríguez, ${ }^{3}$ Joachim Bansmann, ${ }^{4}$ Rocio Yanes, ${ }^{5}$ \\ Ulrich Nowak, ${ }^{5}$ Armin Kleibert, ${ }^{1, *}$ and Frithjof Nolting ${ }^{1}$ \\ ${ }^{1}$ Swiss Light Source, Paul Scherrer Institut, Villigen PSI 5232, Switzerland \\ ${ }^{2}$ Condensed Matter Theory Group, NUM, Paul Scherrer Institut, Villigen PSI 5232, Switzerland \\ ${ }^{3}$ Departament de Física Fonamental and Institut de Nanociència i Nanotecnologia (IN2UB), \\ Universitat de Barcelona, 08028 Barcelona, Spain \\ ${ }^{4}$ Institute of Surface Chemistry and Catalysis, University of Ulm, 89069 Ulm, Germany \\ ${ }^{5}$ Department of Physics, University of Konstanz, 78457 Konstanz, Germany \\ (Received 25 April 2013; revised manuscript received 25 January 2014; published 10 March 2014)
}

\begin{abstract}
$\mathrm{X}$-ray photoemission electron microscopy combined with $\mathrm{x}$-ray magnetic circular dichroism is used to study the magnetic properties of individual iron nanoparticles with sizes ranging from 20 down to $8 \mathrm{~nm}$. While the magnetocrystalline anisotropy of bulk iron suggests superparamagnetic behavior in this size range, ferromagnetically blocked particles are also found at all sizes. Spontaneous transitions from the blocked state to the superparamagnetic state are observed in single particles and suggest that the enhanced magnetic energy barriers in the ferromagnetic particles are due to metastable, structurally excited states with unexpected life times.
\end{abstract}

DOI: 10.1103/PhysRevLett.112.107201

PACS numbers: 75.30.Gw, 68.37.Yz, 75.50.Bb

Metastability is a well-known phenomenon in condensed matter, where energy barriers prevent a system to relax from a higher-energy state to the ground state. Since the barrier heights usually scale with the system size, the preparation of metastable, higher-energy states becomes possible at the nanoscale [1,2]. Such states can be of profound interest when searching for materials with novel properties. For instance, much effort is currently undertaken to find magnetic nanostructures with properties that enable us to overcome the so-called "superparamagnetic limit," which will occur when further reducing the magnetic bit size for future high-density magnetic data storage devices [3-5]. Such applications require a magnetic energy barrier $E_{m}$ that is sufficiently high to prevent thermally driven switching of the magnetization at relevant temperatures (superparamagnetism). The barriers are usually provided by the magnetic anisotropy energy (MAE), which to first order scales with the particle volume. The magnetic relaxation rate $v$ of a small magnet at a given temperature $T$ is given by an Arrhenius law according to $v=v_{0} \exp \left(-E_{m} /\right.$ $\left.k_{B} T\right)$ with the thermal energy $k_{B} T$. The attempt frequency $v_{0}$ depends on temperature, saturation magnetization, and the MAE [6-8]. The corresponding relaxation time is $\tau_{r}=1 / v$.

A direct experimental access to structural higher-energy states and their impact on the magnetic properties is, however, not trivial, particularly when ensemble measurements are considered. Even monodisperse nanomagnet ensembles can show considerable particle-to-particle variations in their properties either due to size effects, interparticle interactions, their individual interfaces with the environment, or surface effects [9]. Experiments with single particle sensitivity enable us to disentangle the different contributions, and thus to distinguish lower or ground state properties from higher-energy states $[10,11]$. Here, we study the magnetization of individual iron $(\mathrm{Fe})$ nanoparticles by magnetic spectromicroscopy. The magnetocrystalline anisotropy of bulk Fe suggests superparamagnetic (SPM) behavior at room temperature (RT). Indeed, such particles have been found experimentally [12]. However, other authors reported enhanced magnetic energy barriers from ensemble measurements, which predict ferromagnetic (FM) behavior in the present size range [13]. Our experiments with single particle resolution seem to resolve these apparently contradictory observations and reveal that SPM and FM nanoparticles can coexist in the present size range. The FM state is metastable and can spontaneously decay towards the SPM state. We attribute this behavior to a structurally excited state with a remarkable lifetime at RT.

The Fe nanoparticles under investigation are generated by an arc cluster ion source [14] and deposited in situ onto Si substrates which are passivated with a native oxide layer. The kinetic energy of the particles prior to the impact on the substrate is smaller than $0.1 \mathrm{eV} /$ atom. Thus, it is anticipated that particle or substrate damage is avoided [15] (cf. Supplemental Material [16]). The distribution of particle sizes $D$ ranges from 8 to $20 \mathrm{~nm}$ as determined from ex situ height measurements using atomic force microscopy [Fig. 1(a)]. Complementary ex situ high-resolution scanning electron microscopy is used to identify well-shaped, noninteracting particles and to exclude agglomerates from the analysis [17]. A unique identification of the same particles in the different microscopes is possible using 


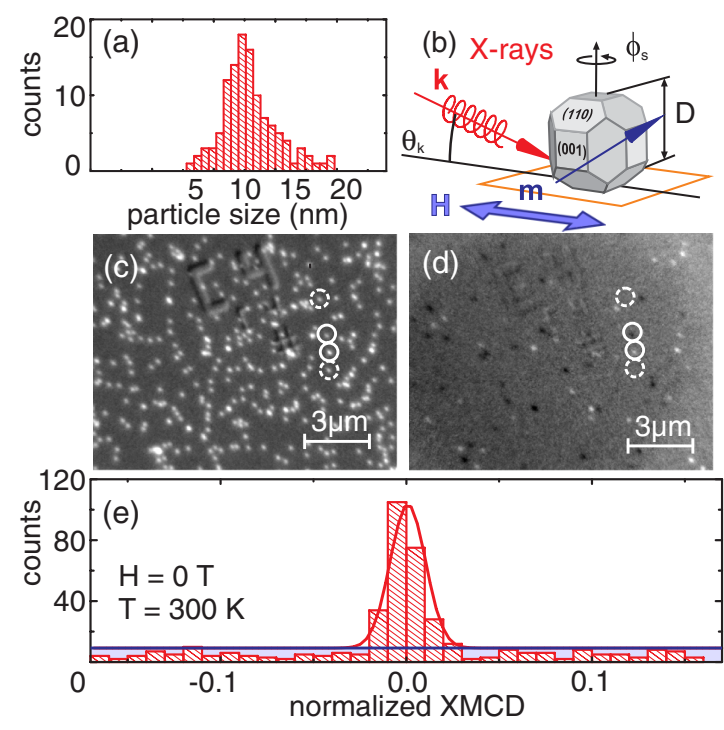

FIG. 1 (color online). (a) Particle size distribution. (b) Sketch of the experimental geometry and the equilibrium shape of the nanoparticles. (c) PEEM elemental contrast image. (d) Magnetic contrast image. Solid (dashed) circles highlight some FM (SPM) particles. (e) Distribution of normalized XMCD contrast values, calculated from the data in (d).

chromium-gold lithographic markers [10]. Particles in the present size range possess a bcc crystal lattice and a saturation magnetization similar to bulk Fe $[14,18,19]$. High-resolution transmission electron microscopy of particles produced with the present cluster source demonstrates a faceted shape close to that predicted by a Wulff construction [14] [cf. Fig. 1(b)]. The deposition results in a nearly random spatial distribution of a few particles per $\mu \mathrm{m}^{2}$ with random crystallographic orientation [18].

The magnetic properties of the particles are studied in situ by means of $\mathrm{x}$-ray photoemission electron microscopy (PEEM) [20]. The experiments are carried out with a pressure below $5 \times 10^{-9}$ mbar. Elemental contrast images [Fig. 1(c)] are obtained by pixelwise division of images recorded successively at the $\mathrm{Fe} L_{3}$ absorption edge $(\sim 709 \mathrm{eV})$ and a pre-edge energy $(\sim 703 \mathrm{eV})$. The magnetization of each particle is visualized using the $\mathrm{x}$-ray magnetic circular dichroism (XMCD) effect [21]. The latter gives rise to a magnetization dependent intensity according to $I\left(C^{ \pm}\right)=I_{0} \pm \gamma(\vec{k} \cdot \vec{m})$, where $I_{0}$ is the isotropic (nonmagnetic) contribution, $\vec{k}$ is the $\mathrm{x}$-ray propagation vector with an angle of incidence $\theta_{k}=16^{\circ}$ [Fig. 1(b)], $\vec{m}$ is the magnetization vector of the particle, $\gamma$ is a material and photon- energy-dependent constant, and $C^{ \pm}$denote circular right- and left-handed polarization. A measure of the magnetization of the particles is given by the normalized XMCD defined as $\left[I\left(C^{+}\right)-I\left(C^{-}\right)\right] /\left[I\left(C^{+}\right)+I\left(C^{-}\right)\right]$.

The experiments reveal that initially about $40 \%$ of the particles show stable magnetic contrast ranging from "white" ( $\vec{m}$ parallel to $\vec{k})$ to "black" ( $\vec{m}$ antiparallel to $\vec{k})$ at RT and under zero external magnetic field [Fig. 1(d)].
These particles have a magnetic relaxation time $\tau_{r}$ much longer than the experimental time resolution $\left(\tau_{\exp }=20 \mathrm{~s}\right)$ and are referred to as FM particles. As discussed below, this magnetically blocked state can be observed over many hours. The other fraction of particles shows no magnetic contrast under these conditions. This is also seen in a histogram of the normalized XMCD contrast of the particles [Fig. 1(e)]. The data reveal a peak at zero XMCD contrast on top of a flat distribution of finite values (indicated by the horizontal line). The latter contribution can be assigned to FM particles with randomly orientated magnetic moments, where the minimum negative and maximum positive XMCD contrast values correspond to "black" or "white" particles in Fig. 1(d). As demonstrated below, the peak at zero can be attributed to SPM particles.

To obtain further insight into the properties of the particle ensemble, an external magnetic field $H$ is applied and the normalized XMCD contrast as a function of $H$ is recorded (Fig. 2). Some representative particles are highlighted in the elemental and magnetic contrast images before applying the magnetic field in Figs. 2(a) and (b). Most of the particles that initially show no magnetic contrast can be magnetized by applying a few mT [cf. particle "A" in Figs. 2(a) and (b) and its magnetization curve in Fig. 2(c)]. This behavior indicates SPM particles with magnetic energy barriers well below that of the FM particles. The latter change their contrast only slightly or switch their contrast at some field values from "white" to "black" or vice versa [cf. particle "B" and its magnetization curve in Fig. 2(d)]. Switching occurs here via thermal excitation, which is promoted by a reduction of $E_{m}$ due to the applied magnetic field. (The Zeeman energy is less than $0.1 \mathrm{eV}$ for the present particles and fields.) The magnetic state of the particles is further confirmed by observing the normalized XMCD as a function of the azimuthal sample orientation $\phi_{s}$ at $H=0 \mathrm{mT}$. The SPM particles show no contrast at any orientation, while the FM particles show an angular dependence according to $\gamma(\vec{k} \cdot \vec{m})$ as shown in the insets of Fig. 2(c) and (d). Correlating the magnetic state with the particle size surprisingly reveals that the particles can be FM or SPM, irrespective of their size (Fig. 3).

To evaluate the magnetic energy barrier of the FM particles, the sample temperature is varied. Most of the FM particles start to alter their magnetic contrast at $340 \mathrm{~K}$ and loose their contrast between 360 and 375 K [Figs. 4(b)-(f)], which indicates that their relaxation time becomes shorter than the experimental time resolution. When subsequently cooling the sample to RT, only about one third of the initially FM particles recover their magnetic contrast. Some of these particles show a contrast reversal, demonstrating that thermally activated fluctuations of the magnetization have occurred during the experiment. With longer observation times thermally induced magnetic switching can be found also at RT [Fig. 4(h)]. The switching occurs between two states with the same XMCD contrast, but opposite sign. 

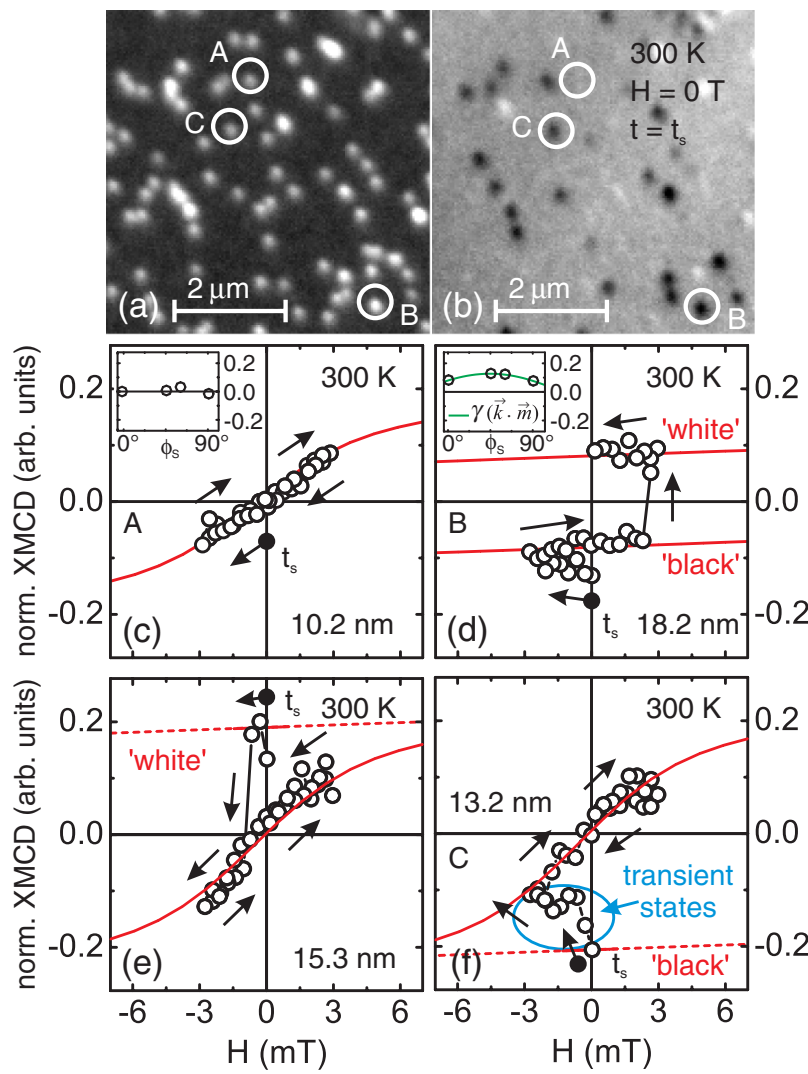

FIG. 2 (color online). (a),(b) Elemental and magnetic contrast images in the initial state before the magnetic field is applied. (c)-(f) Response of individual particles to a sequence of applied magnetic fields $\left(\phi_{s}=0^{\circ}\right)$. The cycle starts with $H=0 \mathrm{mT}$ at time $t_{s}$. The field cycle is indicated by black arrows. The full cycle is recorded over a time of about ten hours. The magnetic response of a particle is given by its field-dependent normalized $\mathrm{XMCD}$ contrast (circles). The red and the dashed lines serve as a guide to the eye. Three particles are denoted as " $A$," " $B$," and " $C$ " in (a) and (b). Their magnetization curves are depicted in (c), (d), and (f). The insets in (c) and (d) show the normalized XMCD recorded as a function of $\phi_{s}(H=0 \mathrm{mT})$. The green line in (d) is a fit to the data. The magnetization curves in (e) and (f) demonstrate spontaneous transitions from (initially "white" and "black") FM states to SPM behavior.

This behavior indicates a $180^{\circ}$ reversal of $\vec{m}$, which is typical for a dominant uniaxial anisotropy contribution. From Figs. 4(b)-(h) we obtain a mean magnetic energy barrier $E_{m} \geq 0.76 \mathrm{eV}$ via the Arrhenius law and an estimate of the corresponding attempt frequency for all particles exhibiting the reversible FM/SPM transition (cf. Supplemental Material [16]).

About two thirds of the initially FM nanoparticles do not recover their magnetic contrast upon heating to $375 \mathrm{~K}$ and subsequent cooling down to RT. Heating the sample to $420 \mathrm{~K}$ leads to an irreversible loss of magnetic contrast in all initially FM particles. Thus, another thermally induced but irreversible relaxation mechanism might exist that significantly alters the magnetic behavior of the particles.

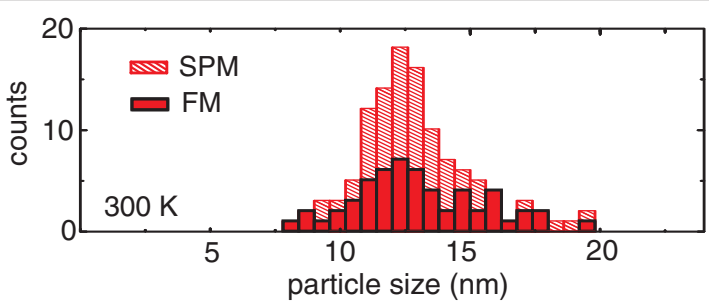

FIG. 3 (color online). The same size distribution as shown in Fig. 1(a), but now the magnetic state of the particles is highlighted with red for FM and shaded for SPM. Particles with a normalized $\mathrm{XMCD} \geq|0.03|$ (with $H=0 \mathrm{mT}$ ) were counted as FM.

Indeed, we observe spontaneous transitions from the FM to the SPM state also at RT as shown, e.g., in Figs. 2(e) and 2(f). These particles are initially ferromagnetically blocked, but after some time their magnetization approaches the response of the SPM particles. The curves further reveal that the transition may evolve via transient states that give rise to some jumps in the magnetization curves.

To assess these observations we estimate the MAE and the related magnetic energy barriers of isolated Fe particles with a bulklike bcc lattice and a highly symmetric shape with (100) and (110) surface facets [Fig. 1(b)]. We refer to this as a structural low-energy state. The total MAE is given by magnetocrystalline, shape, and surface anisotropy contributions. The surface anisotropy energy can be relatively high in $\mathrm{Fe}$ [12], but its contribution to the total magnetic energy barrier can be neglected in the present size range [22].


FIG. 4 (color online). (a) Elemental contrast image. (b)-(f) Evolution of magnetic contrast with temperature. (g) Magnetic contrast upon subsequent cooling to RT. White circles highlight particles that show a reversible FM/SPM transition. Red circles indicate initially FM particles which do not recover their contrast when cooling back to RT. The scale bars in (b)-(g) indicate $2 \mu \mathrm{m}$. (h) Thermally induced fluctuations of the magnetization. (i) Elemental contrast image. (j),(k) Magnetic contrast at RT and at $130 \mathrm{~K}$. Some FM (SPM) particles are highlighted by solid (dashed) circles. 
Also, a highly symmetric Wulff-shaped iron particle has hardly any shape anisotropy contribution [23]. The largest particles studied here $(D=20 \mathrm{~nm})$ consist of about 410,000 atoms. The bulk magnetocrystalline anisotropy constant of $3.3 \mu \mathrm{eV} /$ atom yields then a total MAE of about $E_{\mathrm{MAE}}=$ $1.35 \mathrm{eV}$ [24]. Since the bulk anisotropy is cubic, the associated energy barrier is $E_{m}=E_{\mathrm{MAE}} / 4=0.34 \mathrm{eV}$ [7], which scales with the particle volume. Thus, it is evident that particles in the present size range and with the above defined properties should be SPM at RT (cf. Supplemental Material [16]). Indeed, the observed SPM particles behave this way and their SPM state persists down to $130 \mathrm{~K}$ [Figs. 4(i)-(k)].

Our experiments demonstrate significantly different magnetic properties in the FM particles with a dominant uniaxial anisotropy and a much larger magnetic energy barrier when compared to the SPM particles or the low-energy state discussed above. A uniaxial magnetic anisotropy term could arise due to deviations from the symmetric shape. Assuming for instance a slightly ellipsoidal shape with an aspect ratio of 1.05 , we obtain a magnetic energy barrier of about $E_{m}=0.2 \mathrm{eV}$ for a particle with a volume corresponding to a size of $12 \mathrm{~nm}$ [25]. This value indicates that shape anisotropy may indeed contribute to the properties of the FM particles, but on its own it is not sufficient to explain our observations. For instance for the smallest particles with $D=8 \mathrm{~nm}$, an aspect ratio of 1.5 would be required to yield an FM state at RT. While particles with this geometry would still show a coherent reversal mechanism (not via domain nucleation and propagation) with high magnetic stability [26,27], their presence in our samples can be excluded based on our complementary structural characterization. Alternatively, strain can yield a sizeable magnetoelastic anisotropy contribution [28]. Using the magnetoelastic constants known from bulk Fe, a strain of $\sim 4 \%$ would be required in the $8 \mathrm{~nm}$ particles to explain the observed magnetic stability. However, homogenous strain of this magnitude appears very unlikely to persist in a nanoparticle of this size. This suggests that either the magnetoelastic properties in nanoparticles significantly differ from the bulk or the strain is strongly localized, e.g., via defects or dislocations. Such structural modifications are usually energetically unfavorable, when compared to the low-energy state described above and are therefore often discarded. However, our experiments show FM states with a surprisingly long lifetime, which we attribute to metastable local structural modifications. In fact, we observe that at RT less then $10 \%$ of the FM particles show the transition to SPM behavior during the total time of the experiment of about ten hours.

The direct experimental access to such structural modifications in nanoparticles remains challenging. Only recently have techniques been developed that enable the mapping of strain in supported single nanoparticles. These studies demonstrate that inhomogenous strain may arise due to surface tension and defects at the particle-substrate interface [29]. Moreover, if dislocation structures within the particles were formed either during growth or upon the impact onto the substrate, such dislocations could also result in a uniaxial magnetic anisotropy [30]. Respectively, enhanced magnetic energy barriers in $\mathrm{Fe}$ nanostructures have been reported by several authors [13,23,31,32], while others found bulklike MAE $[9,12,33]$. Our data show that both forms of particles can coexist over a large size range and that the metastable FM particles can transform into a state with magnetic properties closer to the structural low-energy state. This observation further demonstrates the presence of two different but interrelated phenomena with different time scales: one associated with irreversible structural relaxation and the other associated with magnetic switching. Here, the latter time scale changes as a result of the structural relaxation and causes a crossover from FM to SPM behavior. Whether an FM or SPM character is predominantly found in experiments may depend on the sample treatment, e.g., on whether ground state relaxation of the particles has been achieved by thermal annealing or not.

In summary, we have demonstrated that Fe nanoparticles can possess metastable magnetic properties that are significantly different from those derived from the properties of bulk Fe. Rather than all particles in the investigated size range exhibiting the expected superparamagnetism, a significant proportion of the $\mathrm{Fe}$ nanoparticles demonstrate FM behaviour. This FM proportion is characterized by uniaxial magnetization dynamics. The altered magnetic properties are attributed to metastable structurally excited states of the particles which can spontaneously relax to a lower-energy state that exhibits the anticipated SPM behavior. The coexistence of SPM and FM particles with the same size demonstrates that simple scaling of magnetic properties may not reflect the behavior of a nanoparticle ensemble-even when monodisperse, noninteracting samples are studied. Finally, we notice that the uniaxial magnetization dynamics in the FM particles indicate magnetic bistability - a property that is relevant for applications, such as magnetic data storage. Thus, searching for ways to stabilize nonequilibrium structures made from softmagnetic materials such as Fe might provide an interesting alternative to nanoparticles with ground state properties of hard-magnetic bulk alloys.

We thank A. Weber, R. Schelldorfer, J. Honegger, and J. Krbanjevic (Paul Scherrer Institut) for technical support. We appreciate fruitful discussions with C. A. F. Vaz. This work was supported by the Swiss Nanoscience Institute, University of Basel and by the DFG-SPP 1153 (Grant No. BA 1612/3-3). A. F. R. acknowledges support from the MICIIN "Ramón y Cajal" Programme. Part of this work was performed at the Surface/Interface:Microscopy (SIM) beam line of the Swiss Light Source, Paul Scherrer Institut, Villigen, Switzerland. 
*Corresponding author.armin.kleibert@psi.ch

[1] C.-C. Chen, A. B. Herholds, C.S. Johnson, and A. P. Alivisatos, Science 276, 398 (1997).

[2] V. F. Puntes, K. M. Krishnan, and A. P. Alivisatos, Science 291, 2115 (2001).

[3] S. Sun, C. B. Murray, D. Weller, L. Folks, and A. Moser, Science 287, 1989 (2000).

[4] V. Skumryev, S. Stoyanov, Y. Zhang, G. Hadjipanayis, D. Givord, and J. Nogues, Nature (London) 423, 850 (2003).

[5] C. Antoniak, M. E. Gruner, M. Spasova, A. V. Trunova, F. M. Römer, A. Warland, B. Krumme, K. Fauth, S. Sun, P. Entel, M. Farle, and H. Wende, Nat. Commun. 2, 528 (2011).

[6] W. F. Brown, Phys. Rev. 130, 1677 (1963).

[7] I. Einstein and A. Aharoni, Phys. Rev. B 16, 1278 (1977).

[8] U. Nowak, O. N. Mryasov, R. Wieser, K. Guslienko, and R. W. Chantrell, Phys. Rev. B 72, 172410 (2005).

[9] F. Kronast, N. Friedenberger, K. Ollefs, S. Gliga, L. Tati-Bismath, R. Thies, A. Ney, R. Weber, C. Hassel, F. M. Römer, A. V. Trunova, C. Wirtz, R. Hertel, H. A. Dürr, and M. Farle, Nano Lett. 11, 1710 (2011).

[10] A. Fraile Rodríguez, A. Kleibert, J. Bansmann, A. Voitkans, L. J. Heyderman, and F. Nolting, Phys. Rev. Lett. 104, 127201 (2010).

[11] W. Wernsdorfer, E. B. Orozco, K. Hasselbach, A. Benoit, B. Barbara, N. Demoncy, A. Loiseau, H. Pascard, and D. Mailly, Phys. Rev. Lett. 78, 1791 (1997).

[12] F. Bodker, S. Morup, and S. Linderoth, Phys. Rev. Lett. 72, 282 (1994).

[13] J. Carvell, E. Ayieta, A. Garvin, R. Cheng, V. R. Shah, and P. Sokol, J. Appl. Phys. 107, 103913 (2010).

[14] A. Kleibert, J. Passig, K.-H. Meiwes-Broer, M. Getzlaff, and J. Bansmann, J. Appl. Phys. 101, 114318 (2007).

[15] V. N. Popok, I. Barke, E. E. B. Campbell, and K.-H. Meiwes-Broer, Surf. Sci. Rep. 66, 347 (2011).

[16] See Supplemental Material at http://link.aps.org/ supplemental/10.1103/PhysRevLett.112.107201 for further details on the sample preparation and numerical estimates of the relaxation times and energy barriers.
[17] M. Ruano, M. Díaz, L. Martínez, E. Navarro, E. Román, M. García-Hernandez, A. Espinosa, C. Ballesteros, R. Fermento, and Y. Huttel, Phys. Chem. Chem. Phys. 15, 316 (2013).

[18] A. Kleibert, A. Voitkans, and K.-H. Meiwes-Broer, Phys. Rev. B 81, 073412 (2010).

[19] T. Vystavel, G. Palasantzas, S. A. Koch, and J. Th. M. De Hosson, Appl. Phys. Lett. 82, 197 (2003).

[20] L. Le Guyader, A. Kleibert, A. Fraile Rodríguez, S. El Moussaoui, A. Balan, M. Buzzi, J. Raabe, and F. Nolting, J. Electron Spectrosc. Relat. Phenom. 185, 371 (2012).

[21] J. Stöhr, Y. Wu, B. D. Hermsmeier, M. G. Samant, G. R. Harp, S. Koranda, D. Dunham, and B. P. Tonner, Science 259, 658 (1993).

[22] R. Yanes, O. Chubykalo-Fesenko, H. Kachkachi, D. A. Garanin, R. Evans, and R. W. Chantrell, Phys. Rev. B 76, 064416 (2007).

[23] M. Jamet, W. Wernsdorfer, C. Thirion, V. Dupuis, P. Melinon, A. Perez, and D. Mailly, Phys. Rev. B 69, 024401 (2004).

[24] H. P. Klein and E. Kneller, Phys. Rev. 144, 372 (1966).

[25] M. Beleggia, M. De Graef, and Y. Millev, Philos. Mag. 86, 2451 (2006).

[26] H. B. Braun, Phys. Rev. Lett. 71, 3557 (1993).

[27] M. Bode, O. Pietzsch, A. Kubetzka, and R. Wiesendanger, Phys. Rev. Lett. 92, 067201 (2004).

[28] D. Sander, Rep. Prog. Phys. 62, 809 (1999).

[29] M. A. Pfeifer, G. J. Williams, I. A. Vartanyants, R. Harder, and I. K. Robinson, Nature (London) 442, 63 (2006).

[30] D. Pomfret and M. Prutton, Phys. Status Solidi (a) 19, 423 (1973).

[31] D. L. Peng, T. Hihara, K. Sumiyama, and H. Morikawa, J. Appl. Phys. 92, 3075 (2002).

[32] J. P. Pierce, M. A. Torija, Z. Gai, J. Shi, T. C. Schulthess, G. A. Farnan, J. F. Wendelken, E. W. Plummer, and J. Shen, Phys. Rev. Lett. 92, 237201 (2004).

[33] A. V. Trunova, R. Meckenstock, I. Barsukov, C. Hassel, O. Margeat, M. Spasova, J. Lindner, and M. Farle, J. Appl. Phys. 104, 093904 (2008). 